OPEN ACCESS

Edited by:

Daniele Bottai,

University of Milan, Italy

Reviewed by:

Xinyu Zhao,

University of Wisconsin-Madison,

United States

Juan Andres Rubiolo,

University of Santiago

de Compostela, Spain

Ola Hermanson,

Karolinska Institutet (KI), Sweden

*Correspondence:

Sven Wellmann

sven.wellmann@ukbb.ch

Xinzhou Zhu

xinzhou.zhu@unibas.ch

${ }^{t}$ These authors share first authorship

Specialty section:

This article was submitted to

Stem Cell Research,

a section of the journal

Frontiers in Cell and Developmental

Biology

Received: 21 June 2019 Accepted: 04 November 2019

Published: 21 November 2019

Citation:

Yan J, Goerne T, Zelmer A,

Guzman R, Kapfhammer JP,

Wellmann $S$ and Zhu X (2019) The RNA-Binding Protein RBM3 Promotes Neural Stem Cell (NSC) Proliferation

Under Hypoxia.

Front. Cell Dev. Biol. 7:288.

doi: 10.3389/fcell.2019.00288

\section{The RNA-Binding Protein RBM3 Promotes Neural Stem Cell (NSC) Proliferation Under Hypoxia}

\author{
Jingyi Yan ${ }^{1 \dagger}$, Tessa Goerne ${ }^{1 \dagger}$, Andrea Zelmer ${ }^{1}$, Raphael Guzman ${ }^{2}$, \\ Josef P. Kapfhammer ${ }^{2}$, Sven Wellmann ${ }^{1,3 *}$ and Xinzhou Zhu ${ }^{1 *}$
}

' Department of Neonatology, University Children's Hospital Basel (UKBB), Basel, Switzerland, ${ }^{2}$ Department of Biomedicine, University of Basel, Basel, Switzerland, ${ }^{3}$ Department of Neonatology, University Children's Hospital Regensburg (KUNO),

Regensburg, Germany

Neural stem cells (NSCs) reside physiologically in a hypoxic niche to maintain selfrenewal and multipotency. Whereas mild hypoxia is known to promote NSC proliferation, severe hypoxia in pathological conditions exerts the reverse effect. The multi-functional RNA-binding protein RBM3 is abundant in NSCs and can be regulated by hypoxic exposure. Although RBM3 has been shown to accelerate cell growth in many cell types, whether and how it affects NSC proliferation in hypoxic environment remains largely unknown. In this study, we tested how RBM3 regulates cell proliferation under hypoxia in C17.2 mouse NSC cell line and in primary mouse NSCs from both the forebrain of postnatal day 0 (PO) mice and the subgranular zone (SGZ) of adult mice. Our results demonstrated that RBM3 expression was highly sensitive to hypoxia, and NSCs were arrested in G0/G1 phase by 5, 2.5, and $1 \% \mathrm{O}_{2}$ treatment. When we overexpressed RBM3, hypoxia-induced cell cycle arrest in G0/G1 phase was relieved and more cell transit into S phase was observed. Furthermore, cell viability under hypoxia was also increased by RBM3. In contrast, in RBM3-depleted primary NSCs, less BrdUincorporated cells were detected, indicating exacerbated cell cycle arrest in G1 to S phase transition. Instead, overexpressed RBM3 significantly increased proliferation ratio in primary NSCs. Our findings indicate RBM3 as a potential target to maintain the proliferation capacity of NSCs under hypoxia, which can be important in NSC-based therapies of acute brain injury and chronic neurodegenerative diseases.

Keywords: RBM3, CIRP, oxygen, neural stem cell, cell cycle

\section{INTRODUCTION}

Physiological oxygen levels in organisms are considerably lower than ambient oxygen tension (21\%) and vary widely in different tissues to adapt oxygen consumption requirements of diverse cell types (Panchision, 2009; De Filippis and Delia, 2011). Neural stem cells (NSCs) are the main sources to generate neuronal and glial cells of the central nervous system during embryonic and postnatal development and are maintained in specific regions during adulthood for consistent neurogenesis (Gage and Temple, 2013; Bond et al., 2015). They also contribute to neuro-regeneration after acute injuries in the brain or spinal cord (Gage and Temple, 2013; Ludwig et al., 2018). Neurogenic niches, which are the microenvironment for NSC self-renewal and differentiation, contain a relatively wide range of oxygen tension from $<1$ to $8 \%$ (Mohyeldin et al., 2010). The heterogeneity of oxygen 
tension exerts opposite functions on NSC proliferation. While mild hypoxia substantially promotes NSC proliferation, severe hypoxia suppresses their growth and maintains them in quiescent status (Santilli et al., 2010; Felfly et al., 2011). Furthermore, different severity of hypoxia can also have opposite effects on NSC differentiation and neurogenesis (Francis and Wei, 2010; De Filippis and Delia, 2011; Vieira et al., 2011). Therefore, the precise control of hypoxic environment is critical for the maintenance of NSC quiescence/activation status and NSC amount in the pool, as well as for the regulation of their differentiation upon diverse demands.

The appropriate oxygen concentration can be different for in vivo animal studies and for in vitro cell models when investigating NSCs. Traditionally, $21 \% \mathrm{O}_{2}$ is used as the standard laboratory oxygen supply concentration for cell culture (including NSC culture) in vitro, but some researchers questioned whether $21 \% \mathrm{O}_{2}$ results in a relatively hyperoxic environment and may change the physiological characters of NSCs (Clarke and Van Der Kooy, 2009; Yuan et al., 2015; Mas-Bargues et al., 2019). Atmospheric oxygen tension in vitro may lead to a shift of NSC proliferation pattern. Therefore, lower oxygen level can be superior for NSC culture in vitro, when intending to mimic in vivo NSC characters. Instead, $8 \% \mathrm{O}_{2}$ is considered as physiological oxygen tension in neurogenic niche, $2.5 \% \mathrm{O}_{2}$ is considered as moderate hypoxia, and $1 \% \mathrm{O}_{2}$ is considered as severe hypoxia (Panchision, 2009; De Filippis and Delia, 2011).

The multi-functional RNA-binding protein RBM3 is typically inducible by cold exposure (Danno et al., 2000; Zhu et al., 2016). Besides cold stress, RBM3 responds to hypoxia as well (Wellmann et al., 2004). During development, RBM3 expression is abundant in neurogenic niches and co-localizes with NSC marker nestin (Pilotte et al., 2009). RBM3 has been recently reported to promote neurogenesis via Yap during embryonic stage (Xia et al., 2018). Other studies also suggest that RBM3 plays an important role in the proliferation of cancer cells, fibroblasts, and HEK293 cells (Sureban et al., 2008; Wellmann et al., 2010; Matsuda et al., 2011; Chen et al., 2019). Besides, in recent years, a series of studies have demonstrated that RBM3 can promote the survival of neuroblastoma cells, which are widely used to replace NSCs in neuronal differentiation assays in vitro, upon diverse stressful treatments (Yang et al., 2017; Zhuang et al., 2017; Ushio and Eto, 2018). However, it remains unclear how RBM3 regulates NSC proliferation under hypoxic conditions.

In this study, we investigated whether RBM3 expression is affected under hypoxic conditions and elucidated the role of RBM3 in the regulation of cell cycle in mouse NSC cell line and primary murine NSCs exposed to hypoxia.

\section{MATERIALS AND METHODS}

\section{Animals}

The research protocol, approved by the Cantonal Veterinary Office of Basel, was conducted according to the Ethical Principles and Guidelines for Experiments on Animals of the Swiss Academy of Medical Sciences and the Swiss Academy of Sciences. RBM3 wild type (WT) and knockout (KO) mice with C57BL/6 background were generated by Prof. Tadatsugu Taniguchi (University of Tokyo, Japan) (Matsuda et al., 2011) and were kindly provided by his group. Since $R B M 3$ is $\mathrm{X}$-chromosome gene, only male mice were used in this study.

\section{Cell Isolation and Culture}

Primary NSCs were isolated from the whole brain excluding cerebellum of postnatal day 0 (P0) mice or from the subgranular zone (SGZ) of 2-month-old adult mice as described previously (Zhu et al., 2019). Briefly, the forebrains from P0 mice or the dentate gyrus from adult mice were dissociated with papain (Worthington) and DNase I (Sigma) and then undissociated cell clusters were removed by a cell strainer (Sigma). Dissociated cells were cultured in serum-free DMEM-F12 (Gibco) supplemented with B27 supplement (Gibco), $2 \mathrm{mM} \mathrm{L-glutamine} \mathrm{(Gibco),}$ 20 ng/ml EGF (PeproTech), and 20 ng/ml FGF2 (PeproTech). After glial cells and neurons died, primary NSCs were maintained as neurospheres in uncoated dishes.

C17.2 mouse NSC line was purchased from Sigma and cultured in DMEM (Gibco) supplemented with 10\% FBS (Gibco) and $2 \mathrm{mM}$ L-glutamine (Gibco).

\section{Plasmid Transient Transfection}

pCEP4 mammalian expression vector was purchased from ThermoFisher Scientific. rbm3 gene was cloned into pCEP4 vector in our previous work for exogenous overexpression (Chip et al., 2011). The empty vector or RBM3-overexpressing vector was transiently transfected into C17.2 cells by electroporation with Cell Line Nucleofector Kit V (Lonza) using the Nucleofector I device (Lonza). For transfections in primary NSCs, cells were first dissociated from neurospheres to single cells by Trypsin (Sigma) and then transfected with DNA vectors using the Mouse Neural Stem Cell Nucleofector Kit (Lonza) and the Nucleofector I device (Lonza).

\section{Hypoxia Exposure}

Before hypoxic treatment, primary NSCs in the form of neurospheres were dissociated into single cells by Trypsin (Sigma) and seeded into poly-L-lysine pre-coated 16-well chamber slides (Labtek) at a density of $1 \times 10^{4} /$ well as monolayer culture in ambient normoxic condition $\left(21 \% \mathrm{O}_{2}\right)$. For transfected primary NSCs, additional overnight recovery in uncoated 12well plate was required before seeding to chamber slides. After $24 \mathrm{~h}$ (for non-transfected NSCs) or $48 \mathrm{~h}$ (for transfected NSCs) growth, $20 \mu \mathrm{M}$ BrdU was added into the medium and the slides were transferred to a hypoxic incubator (MiniGalaxy A, RS Biotech) with indicated oxygen levels. Slides in an ambient normoxic incubator served as a control group.

Non-transfected and transfected C17.2 cells were seeded either in six-well plates or in 15-cm-diameter dishes, and transferred to a hypoxic incubator with indicated oxygen levels. Plates or dishes in ambient normoxic incubator served as a control group.

\section{RNA Isolation and Real-Time PCR}

Total RNA were purified from $2 \times 10^{5}$ cultured cells by the ReliaPrep RNA Cell Miniprep System (Promega). cDNA was 
synthesized from $1 \mu \mathrm{g}$ total RNA using the GoScript Reverse Transcription System (Promega). Real-time PCR was performed in $15 \mu \mathrm{l}$ volume with the GoTaq qPCR System (Promega) on the CFX Connect Real-Time PCR Detection System (Bio-Rad). The PCR cycle was run as follows: pre-denaturation by $95^{\circ} \mathrm{C}$ for $5 \mathrm{~min}$, and then $95^{\circ} \mathrm{C}$ for $15 \mathrm{~s}$ and $60^{\circ} \mathrm{C}$ for $1 \mathrm{~min}$ for 40 cycles. To calculate relative gene expression, the $2^{-\Delta \Delta C T}$ method was used. Primer sequences for real-time PCR are listed below. To correct for differences in both RNA quality and quantity between samples as well as changes in oxygen exposure, six housekeeping genes were used, namely, beta-actin $(a c t b)$, glyceraldehyde 3-phosphate dehydrogenase (gapdh), ribosomal protein L13a ( $r p l 13 a), 45 \mathrm{~S}$ pre-ribosomal RNA ( $r n 45 s), 28 \mathrm{~S}$ ribosomal RNA ( $r n 28 s 1)$, and alpha-tubulin-1 (tuba1):

Mouse-rbm3-forward: 5' CTT CAG CAG CTT TGG GCC TA $3^{\prime}$

Mouse-rbm3-reverse: $5^{\prime}$ CCC ATC CAG GGA CTC TCC AT $3^{\prime}$

Mouse-cirp-forward: 5' CCA AGT ATG GGC AGA TCT CCG A $3^{\prime}$

Mouse-cirp-reverse: 5' CTG CCG CCC GTC CAC AGA CT $3^{\prime}$

Mouse- $k d m 3 a$-forward: $5^{\prime}$ GAG CTG TTT CCC ACA CCG A $3^{\prime}$

Mouse- $k d m 3 a$-reverse: $5^{\prime}$ TGC TTT TCT CTG AAG GCT $3^{\prime}$

Mouse- $a c t b$-forward: $5^{\prime}$ GGC CAA CCG TGA AAA GAT GA 3'

Mouse- $a c t b$-reverse: $5^{\prime}$ CAC AGC CTG GAT GGC TAC GT $3^{\prime}$

Mouse-gapdh-forward: 5' AAC GAC CCC TTC ATT GAC $3^{\prime}$

Mouse-gapdh-reverse: $5^{\prime}$ TCC ACG ACA TAC TCA GCA C $3^{\prime}$

Mouse-rpl13a-forward: 5' GCG GAT GAA TAC CAA CCC $3^{\prime}$

Mouse-rpl13a-reverse: 5' GTA GGC TTC AGC CGA ACA AC $3^{\prime}$

Mouse-rn45s-forward: $5^{\prime}$ GTA ACC CGT TGA ACC CCA TT $3^{\prime}$

Mouse-rn45s-reverse: $5^{\prime}$ CCA TCC AAT CGG TAG TAG CG $3^{\prime}$

Mouse-rn28s1-forward: 5' TTG AAA ATC CGG GGG AGA G 3'

Mouse-rn28s1-reverse: $5^{\prime}$ ACA TTG TTC CAA CAT GCC AG $3^{\prime}$

Mouse-tuba1-forward: 5' ACA GGA TTC GCA AGC TGG C $3^{\prime}$

Mouse-tuba1-reverse: 5' CCA AGA AGC CCT GGA GAC C $3^{\prime}$

\section{Protein Isolation and Western Blot}

Total proteins were extracted from cultured cells or homogenized mouse brain with lysis buffer (1\% Triton X-100, $50 \mathrm{mM}$ Tris, $150 \mathrm{mM} \mathrm{NaCl}, 1 \times$ Roche Protease Inhibitor Cocktail, $\mathrm{pH}$ 8.0). Total protein concentrations in cleared cell lysates were determined with RC DC Protein Assay (Bio-Rad). Lysates were loaded onto Mini-Protean TGX pre-cast gels (Bio-Rad) and transferred to PVDF membranes (Amersham/GE Healthcare Life Sciences). Membranes were incubated with primary antibodies overnight at $4^{\circ} \mathrm{C}$ and then with HRP-linked secondary antibodies for $1 \mathrm{~h}$ at room temperature (RT).

Primary and secondary antibodies:

Anti-mouse RBM3 (Proteintech, 14363-1-AP): rabbit polyclonal, 1:1000 diluted

Anti-mouse CIRP (Proteintech, 10209-1-AP): rabbit polyclonal, 1:1000 diluted

Anti-mouse GAPDH (Abeam, ab8245): mouse monoclonal, 1:2000 diluted

HRP-linked anti-rabbit secondary antibody (Cell Signaling Technology, 7074S): 1:5000 diluted

HRP-linked anti-mouse secondary antibody (Cell Signaling Technology, 7076S): 1:5000 diluted.

Band relative quantification was performed by Image J $(\mathrm{NIH})$. Each band was outlined and the mean grayscale value was measured. Background intensity was subtracted from the measured values. The ratios of target proteins (RBM3 and CIRP) and loading control (GAPDH) were calculated.

\section{Flow Cytometry}

Flow cytometry analysis was performed with C17.2 cells on FACSCANTO II device (BD Biosciences). After hypoxic treatment, cells were fixed with ethanol for viability and cell cycle analysis. Cell viability was measured with $1 \times 10^{6}$ cells using LIVE/DEAD Viability/Cytotoxicity Kit (ThermoFisher Scientific). For cell cycle analysis, $1 \times 10^{6}$ cells were analyzed with propidium iodide (Sigma).

\section{Immunofluorescent Staining}

After hypoxic treatment, cells in chamber slides were fixed 4\% paraformaldehyde for $10 \mathrm{~min}$ at RT. For BrdU staining, fixed cells were treated $2 \mathrm{M} \mathrm{HCl}$ for $30 \mathrm{~min}$ and neutralized with $0.1 \mathrm{M}$ sodium borate ( $\mathrm{pH}$ 8.5) for $10 \mathrm{~min}$ before blocking. For permeabilization and blocking, cells were treated with $0.5 \%$ Triton X-100 and 5\% normal goat serum in phosphate buffer for $1 \mathrm{~h}$ at RT. Cells were incubated with primary antibodies overnight at $4^{\circ} \mathrm{C}$ and then with Alexa Fluor dye-conjugated secondary antibodies for $2 \mathrm{~h}$ at RT. Nuclei were counterstained with DAPI for 15 min at RT. Images were acquired with an Olympus AX70 fluorescent microscope equipped with a Spot Insight digital camera. For cell quantification, images of five random fields in each experiment were captured under $20 \times$ magnifications, and the total numbers of BrdU + and DAPI + cells in each image were counted. The average percentage of BrdU + /DAPI + cells from five images was used to represent the value of one experiment. Three independent experiments were performed.

Primary antibodies:

Anti-Nestin (Novus, NBP1-02419), rabbit polyclonal, 1:200 diluted

Anti-Sox2 (R\&D, MAB2018), mouse monoclonal, 1:250 diluted 
Anti-Dcx (Millipore, AB2253), guinea pig polyclonal, 1:1000 diluted

Anti-Tuj1 (Tubulin III) (Millipore, AB1637), mouse monoclonal, 1:250 diluted

Anti-BrdU (Abcam, ab6326), rat monoclonal, 1:250 diluted.

\section{Statistical Analysis}

All experiments were repeated three times independently. Quantification data are presented in standard error of the mean (SEM). Statistical analysis was performed by GraphPad Prism 8.0. To compare two groups with a single factor, unpaired $t$ test was used. To compare more than two groups with a single factor, statistical significance was determined by one-way ANOVA followed by Dunnett's multiple comparison. To compare two groups and two factors, statistical significance was determined by two-way ANOVA followed by Tukey's multiple comparison. $p$ value less than 0.05 was reported as significant difference. N.S., not significant; ${ }^{*} p<0.05 ;{ }^{* *} p<0.01$.

\section{RESULTS}

\section{RBM3 Expression Is Sensitively Inhibited by Hypoxia in NSC}

C17.2 mouse NSC line was used for this study, as it harbors NSC properties and is widely investigated with hypoxic treatments (Felfly et al., 2011; Wang et al., 2014; Zhang et al., 2017). We first confirmed the NSC property of C17.2 cells by immunostaining with specific markers. C17.2 cells express NSC marker nestin and sex determining region Y-box 2 (Sox2), but not neuroblast marker doublecortin (Dcx) and neuronal marker neuron-specific Class III $\beta$-tubulin (Tuj1) (Figure 1A), indicating their properties as undifferentiated NSCs.

To test how different oxygen levels influenced RBM3 expression, we exposed C17.2 cells to ambient normoxia (21\% $\mathrm{O}_{2}$ ), very mild hypoxia (18 and $16 \% \mathrm{O}_{2}$ ), mild hypoxia (12 and $8 \% \mathrm{O}_{2}$ ), moderate hypoxia $\left(5\right.$ and $\left.2.5 \% \mathrm{O}_{2}\right)$, and severe hypoxia $\left(1 \% \mathrm{O}_{2}\right)$. Beta-actin gene $(a c t b)$ was selected as reference gene for real-time PCR, as it showed the highest stability under hypoxic conditions among six commonly used housekeeping genes (Supplementary Table S1). The mRNA level of $r b m 3$ was remarkably suppressed even under very mild hypoxic condition, and remained suppressed with mild, moderate, and severe hypoxia (Figure 1B). Notably, at $2.5 \% \mathrm{O}_{2}, r b m 3$ expression was recovered to some extent, but remained lower than ambient normoxic condition (Figure 1B). $k d m 3 a$, a well-characterized hypoxia-inducible gene, was used as positive control (Wellmann et al., 2008), and its expression was upregulated by moderate to severe hypoxia (Figure 1D). At $2.5 \% \quad \mathrm{O}_{2}$ level, $k d m 3 a$ expression reached the peak (Figure 1D). Interestingly, the only known vertebrate homolog of RBM3, cold inducible RNAbinding protein (CIRP) (Zhu et al., 2016), showed a similar reduction of mRNA as rbm3 under hypoxic conditions, but not as sensitive as $r b m 3$ at $18 \% \mathrm{O}_{2}$ level (Figure 1C). Consistently, RBM3 and CIRP protein expressions were also downregulated by $2.5 \%$ hypoxia (Figures 1E,F). In general, our results show that
RBM3 expression is extremely sensitive to hypoxic exposure in C17.2 mouse NSC line.

\section{Moderate to Severe Hypoxia Inhibits C17.2 Cell Proliferation}

In general, NSCs are exposed to moderate hypoxia physiologically and to severe hypoxia pathologically in vivo (De Filippis and Delia, 2011). Here, we evaluated how different degrees of moderate to severe hypoxia influenced the cell cycle of C17.2 cells. Cells were treated with $21,5,2.5$, and $1 \% \mathrm{O}_{2}$ for $24 \mathrm{~h}$, and then stained with propidium iodide and analyzed for cell cycle change by flow cytometry. We observed that moderate $\left(5\right.$ and $\left.2.5 \% \mathrm{O}_{2}\right)$ and severe hypoxia $\left(1 \% \mathrm{O}_{2}\right)$ lead to a remarkable cell cycle arrest, with increased cell number in G0/G1 phase and decreased cell number in $S$ phase (Figure 2). Cell numbers in $\mathrm{S}$ phase and $\mathrm{G} 2 / \mathrm{M}$ phase were reduced in all hypoxic groups (Figure 2). In accordance to a previous report, our data supported the idea that severe hypoxia $\left(1 \% \mathrm{O}_{2}\right)$ inhibited cell cycle in C17.2 cells (Zhang et al., 2017). However, with moderate hypoxia (5 and $2.5 \% \mathrm{O}_{2}$ ), we observed an inhibitory effect as well (Figure 2) rather than a stimulating effect on NSC proliferation (Zhang et al., 2017). As moderate hypoxia (2.5\% $\mathrm{O}_{2}$ ) demonstrated more cell arrest in $\mathrm{S}$ phase than 5 and $1 \%$ hypoxia (Figure 2), and a recovery of expression was observed (Figure 1B), in the following RBM3-overexpressing experiments, we selected this oxygen concentration to examine the function of RBM3 on NSC proliferation and survival.

\section{Overexpression of RBM3 Rescues C17.2 Cell From Hypoxia-Induced Cell Cycle Arrest}

To explore the role of RBM3 in hypoxia-induced cell cycle arrest, we overexpressed RBM3 in C17.2 cells by transient transfection (Figure 3A) before exposing them to moderate hypoxia $\left(2.5 \% \mathrm{O}_{2}\right)$. As RBM3 was found to be cytoprotective (Yang et al., 2017; Zhuang et al., 2017; Ushio and Eto, 2018), we examined the viability of NSCs after moderate hypoxic treatment. Forced RBM3 expression significantly enhanced live cell proportion and reduced dead cell proportion (Figure 3B). While cells were arrested in G0/G1 phase after hypoxic treatment, RBM3 overexpression caused the cells to overcome the arrest (Figure 3C). These data provide evidence that RBM3 positively regulates NSC growth under moderate hypoxic condition by promoting $\mathrm{G} 1$ to $S$ phase transition.

\section{RBM3 Positively Regulates Primary NSC Proliferation Under Hypoxia}

To further confirm the role of RBM3, we took advantage of primary NSCs isolated from the forebrain of postnatal day 0 (P0) RBM3 WT and KO mice. Similar to C17.2 cells, both WT and KO NSCs express NSC marker nestin and Sox2, but neither the neuroblast marker Dcx nor the neuronal marker Tuj1 (Figure 4A). RBM3 depletion in KO NSCs was confirmed by Western blot (Figure 4B). We further treated primary NSCs with ambient normoxia $\left(21 \% \mathrm{O}_{2}\right)$, moderate hypoxia (5 and $2.5 \%$ $\left.\mathrm{O}_{2}\right)$, or severe hypoxia $\left(1 \% \mathrm{O}_{2}\right)$, and labeled cells by $\mathrm{BrdU}$, a 
A

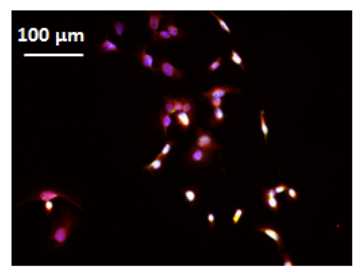

Nestin Sox2 DAPI

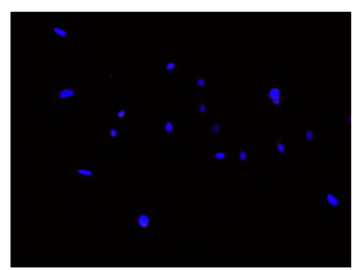

Dcx Tuj1 DAPI

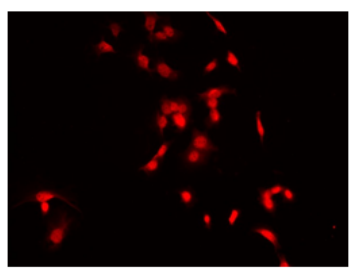

Nestin

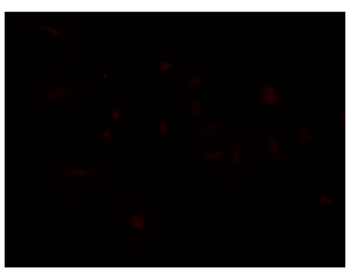

Dcx

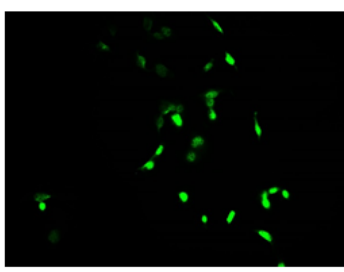

Sox 2

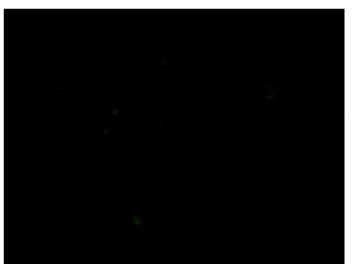

Tuj1

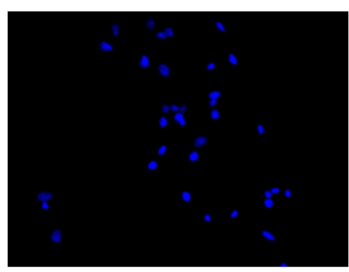

DAPI

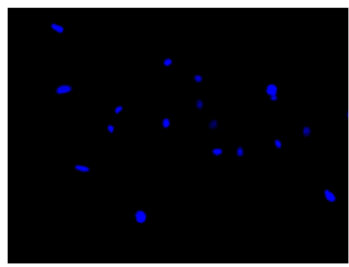

DAPI
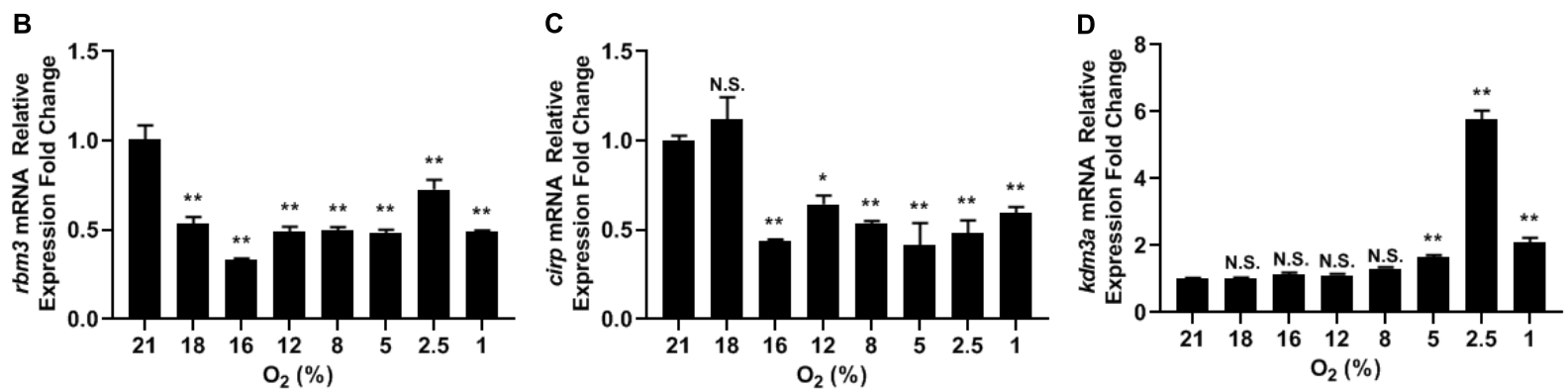

E

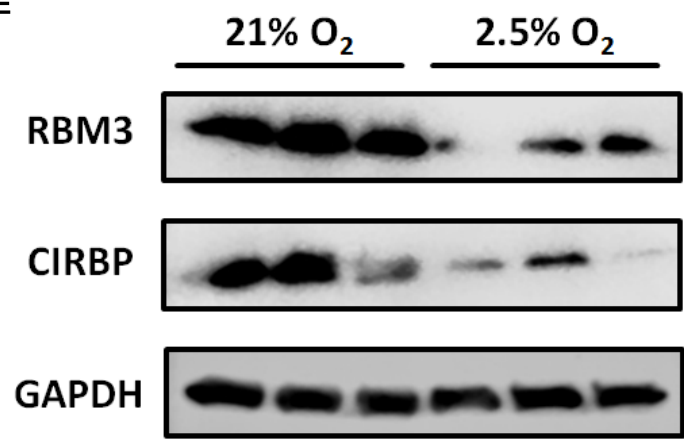

$\mathbf{F}$

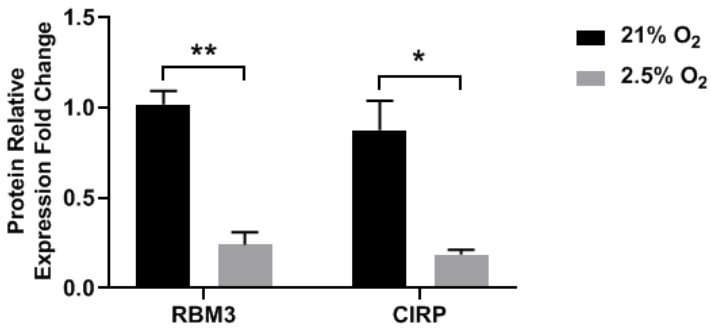

FIGURE 1 | Oxygen-sensitive gene expression in NSCs upon hypoxic exposure. (A) Representative Nestin/Sox2 and Dcx/Tuj1 double stainings of C17.2 cells cultured in standard condition with $21 \% \mathrm{O}_{2}$; nuclei were counterstained with DAPI. (B-D) mRNA expression of rbm3 (B), cirp (C), and kdm3a (D) was measured $16 \mathrm{~h}$ after ambient normoxic $\left(21 \% \mathrm{O}_{2}\right)$ or indicated hypoxic treatment. Actb was used as an internal control. One-way ANOVA followed by Dunnett's test was used to compare each hypoxic condition to the normoxic group. N.S. not significant; ${ }^{*} p<0.05 ;{ }^{* *} p<0.01$. (E) Representative Western blot, RBM3, and CIRP were measured $24 \mathrm{~h}$ after ambient normoxic $\left(21 \% \mathrm{O}_{2}\right)$ or moderate hypoxic $\left(2.5 \% \mathrm{O}_{2}\right)$ treatment; GAPDH was used as a loading control. (F) Band relative intensity in panel (E) was normalized, and unpaired $t$ test was used for statistical analysis. N.S., not significant; ${ }^{*} p<0.05 ;{ }^{* *} p<0.01$.

commonly used proliferation marker for $\mathrm{S}$ phase (Lengronne et al., 2001). After $24 \mathrm{~h}$ incorporation with BrdU, a significant decrease of proliferation ratio ( $\%$ of BrdU + /DAPI + cells) from ambient normoxia $\left(21 \% \mathrm{O}_{2}\right)$ to severe hypoxia $\left(1 \% \mathrm{O}_{2}\right)$ was detected, but not from ambient normoxia $\left(21 \% \mathrm{O}_{2}\right)$ to moderate hypoxia (5 and $2.5 \% \mathrm{O}_{2}$ ) (Figures 4C,D), probably due to the robustness of P0 NSCs with high proliferation rate and resistance to hypoxic stress. The proliferation ratio was remarkably downregulated in KO NSCs when comparing to WT NSCs under ambient normoxic condition $\left(21 \% \mathrm{O}_{2}\right)$ or moderate hypoxic conditions (5 and $2.5 \% \mathrm{O}_{2}$ ), but not significant under severe hypoxic conditions $\left(1 \% \mathrm{O}_{2}\right)$ (Figures 4C,D). In addition, 


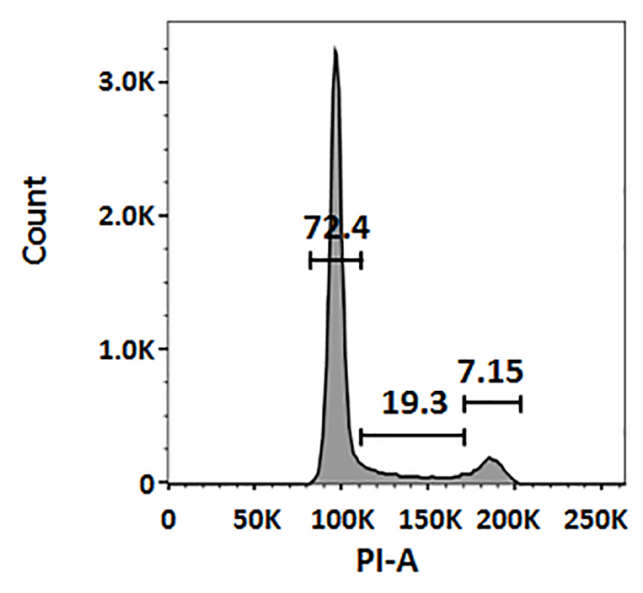

$21 \% \mathrm{O}_{2}$

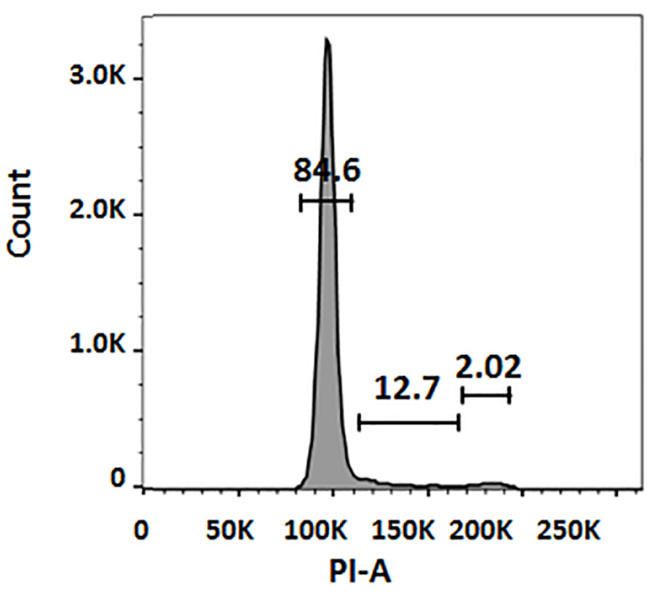

$2.5 \% \mathrm{O}_{2}$

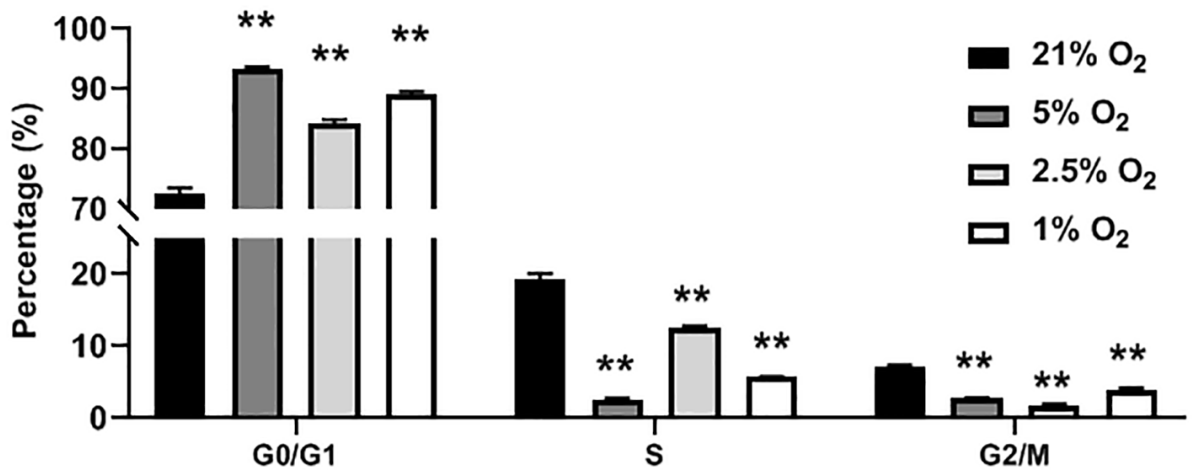

FIGURE 2 | Hypoxia induces cell cycle arrest in NSCs. Cell cycle analysis of C17.2 cells after $24 \mathrm{~h} 5 \% \mathrm{O}_{2}, 2.5 \% \mathrm{O}_{2}$, and $1 \% \mathrm{O}_{2}$ hypoxic treatment. Ambient normoxic $\left(21 \% \mathrm{O}_{2}\right)$ was used as control. Representative flow cytometry DNA histogram with propidium iodide (PI) staining. One-way ANOVA followed by Dunnett's test was used for statistical analysis in G0/G1 phase, S phase, and G2/M phase $(n=3)$. N.S., not significant; ${ }^{* *} p<0.01$.

we overexpressed exogenous RBM3 in primary P0 NSCs by electroporation (Figure 4B). Electroporation-based transfection significantly reduced proliferation ratio to around $70 \%$ under
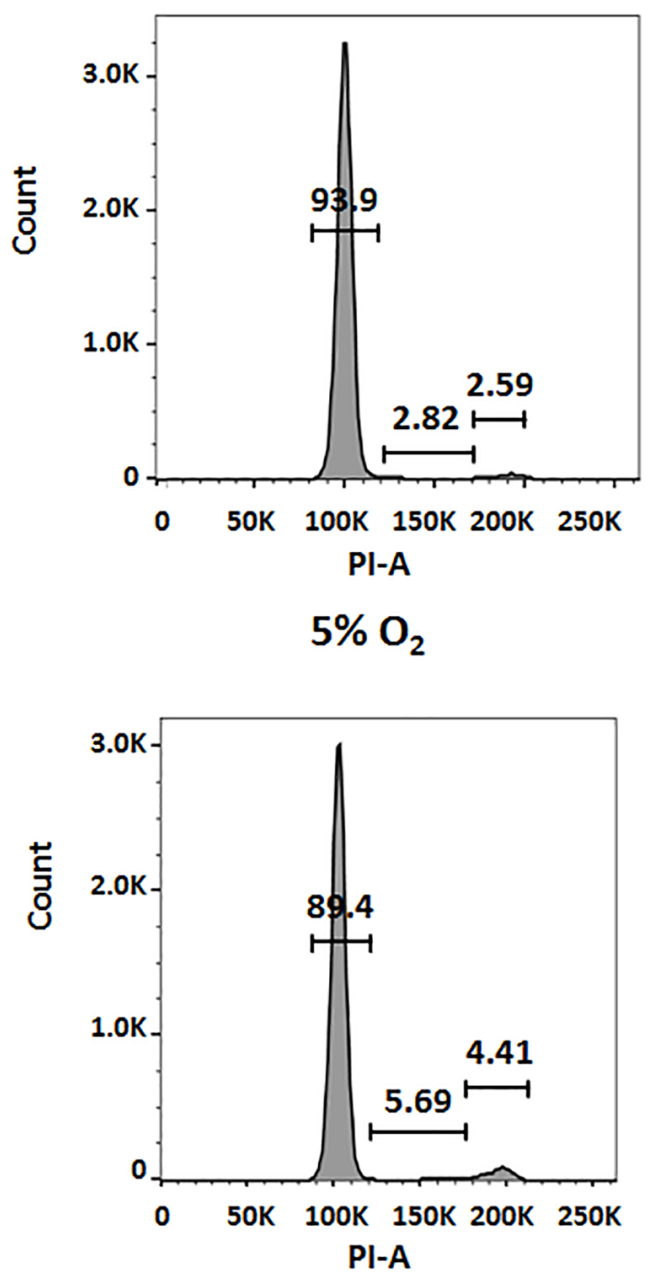

$1 \% \mathrm{O}_{2}$

normoxic conditions $\left(21 \% \mathrm{O}_{2}\right)$, while both moderate hypoxia (5 and $\left.2.5 \% \mathrm{O}_{2}\right)$ and severe hypoxia $\left(1 \% \mathrm{O}_{2}\right)$ further inhibited proliferation ratio remarkably (Figures $4 \mathrm{E}, \mathrm{F}$ ). In contrast to 


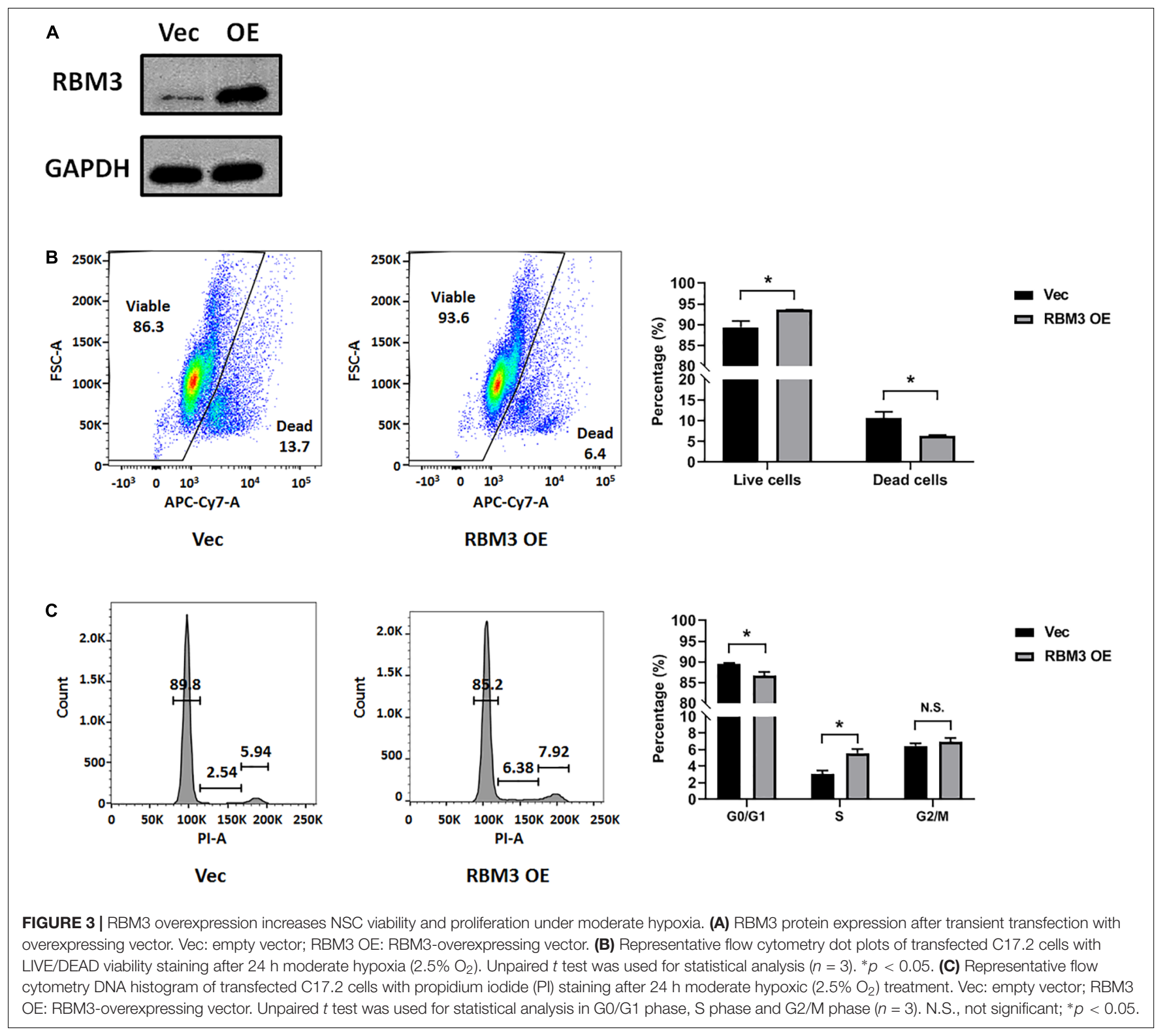

depletion, forced RBM3 expression rescued hypoxia-induced proliferation inhibition partially, but did not alter proliferation under normoxia $\left(21 \% \mathrm{O}_{2}\right)$ (Figures 4E,F).

To confirm that the effect of RBM3 in NSC proliferation is general, we isolated NSCs from the SGZ of adult mice, one of the two well-characterized neurogenic niches in adults (Fuentealba et al., 2012). We did not use subventricular zone (SVZ)-derived NSCs because our previous study showed that RBM3 plays a less important role in the proliferation of SVZNSCs than SGZ-NSCs after hypoxic-ischemic (HI) injury (Zhu et al., 2019). The characters of SGZ-derived NSCs were confirmed by positive staining of nestin/Sox2 and negative staining of Dcx/Tuj1 (Figure 5A). We also confirmed RBM3 depletion in KO SGZ-NSCs and RBM3 overexpression in electroporationtransfected WT SGZ-NSCs (Figure 5B). We also observed a reduction of proliferation of SGZ-NSC in the absence of RBM3 under hypoxia (Figures 5C,D). On the other hand, overexpressed RBM3 elevated SGZ-NSC proliferation ratio under hypoxic conditions (Figures 5E,F).

\section{DISCUSSION}

In this study, we exposed murine NSCs to various degrees of hypoxia and noticed a remarkable downregulation of RBM3 even at very mild hypoxia $\left(18 \% \mathrm{O}_{2}\right)$ with little further change at lower oxygen levels (Figure 1B). The sensitivity of RBM3 expression to hypoxic response is higher than its homolog CIRP (Figures 1B,C). Such a high sensitivity of RBM3 has also been reported in response to environmental temperature change, more than CIRP. Even a $1^{\circ} \mathrm{C}$ change from 37 to $36^{\circ} \mathrm{C}$ is sufficient to stimulate $\mathrm{RBM} 3$ expression in primary 


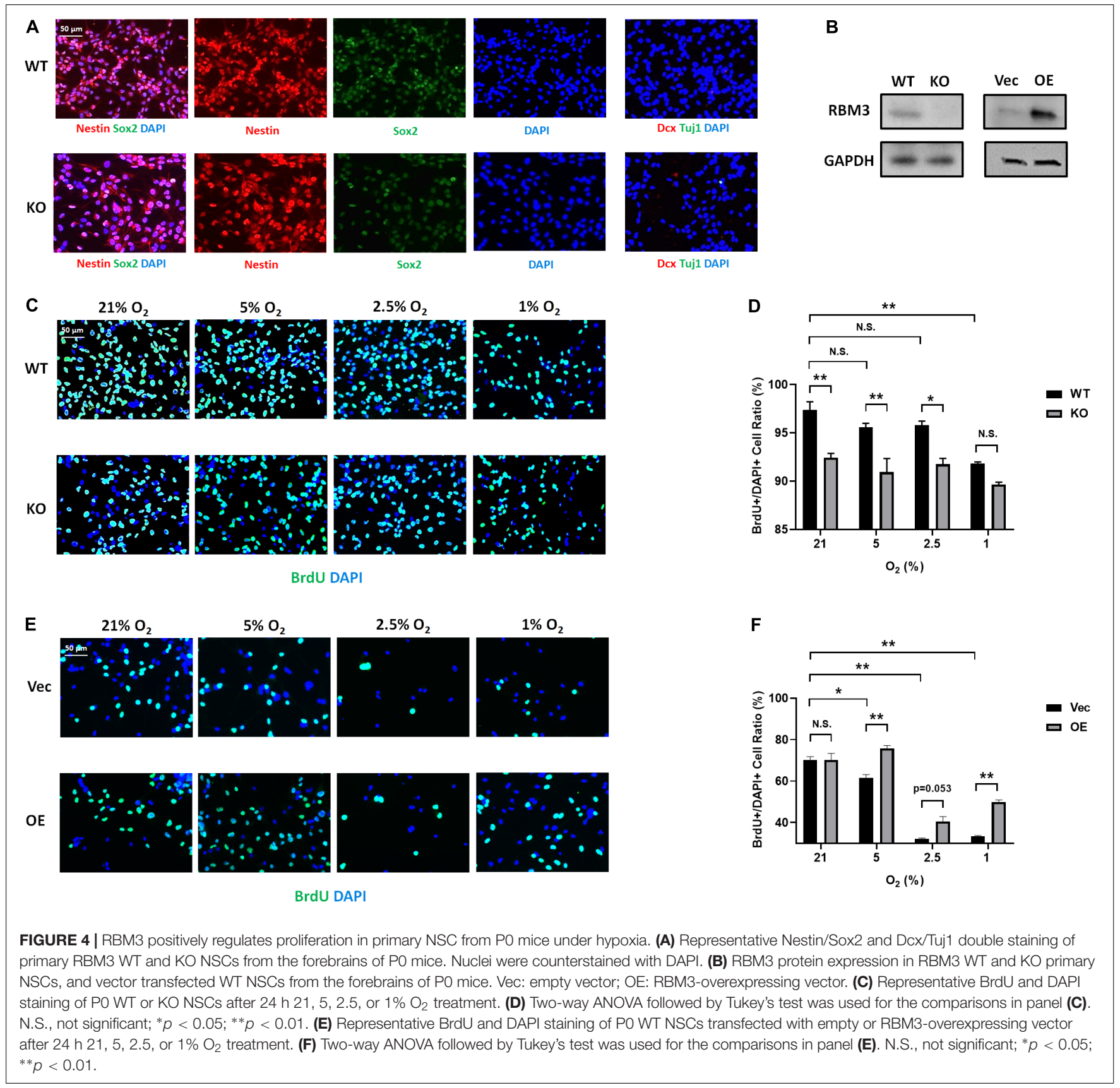

cortical neurons and astrocytes (Jackson et al., 2015). Regarding hypoxia, CIRP expression was also reported to decrease in moderate (3\%) and severe (1\%) hypoxic conditions (Zhang et al., 2017). However, RBM3 was previously found to be induced by moderate $(5 \%)$ and severe $(1 \%)$ hypoxia in HeLa and Hep3B cancer cells (Wellmann et al., 2004). The discrepancy in different cell types indicates that the subtle regulation patterns of the two cold-inducible RNA-binding proteins RBM3 and CIRP by hypoxia can be cell type specific and may involve different regulatory mechanisms. Indeed, cancer stem cells reside in a more hypoxic niche than NSCs and utilize oxygen in different signaling pathways compared to NSCs, in the maintenance of their stemness (Panchision, 2009; Mohyeldin et al., 2010). Notably, in C17.2 cell line, both RBM3 and CIRP are downregulated under moderate to severe hypoxic conditions (Figures 1B,C; Zhang et al., 2017), and both proteins promote G1/S transition in the cell cycle of NSCs upon hypoxic exposure (Figure 3C; Zhang et al., 2017), in accordance with their homogeneous structure and functions as reviewed before (Zhu et al., 2016). We additionally demonstrated consistent effects of RBM3 on NSC proliferation in neonatal and adult primary NSCs (Figures 4, 5). Based on these functions, both RBM3 and CIRP are suggested for potential prevention of hypoxiainduced brain injury. However, considering that CIRP is also 


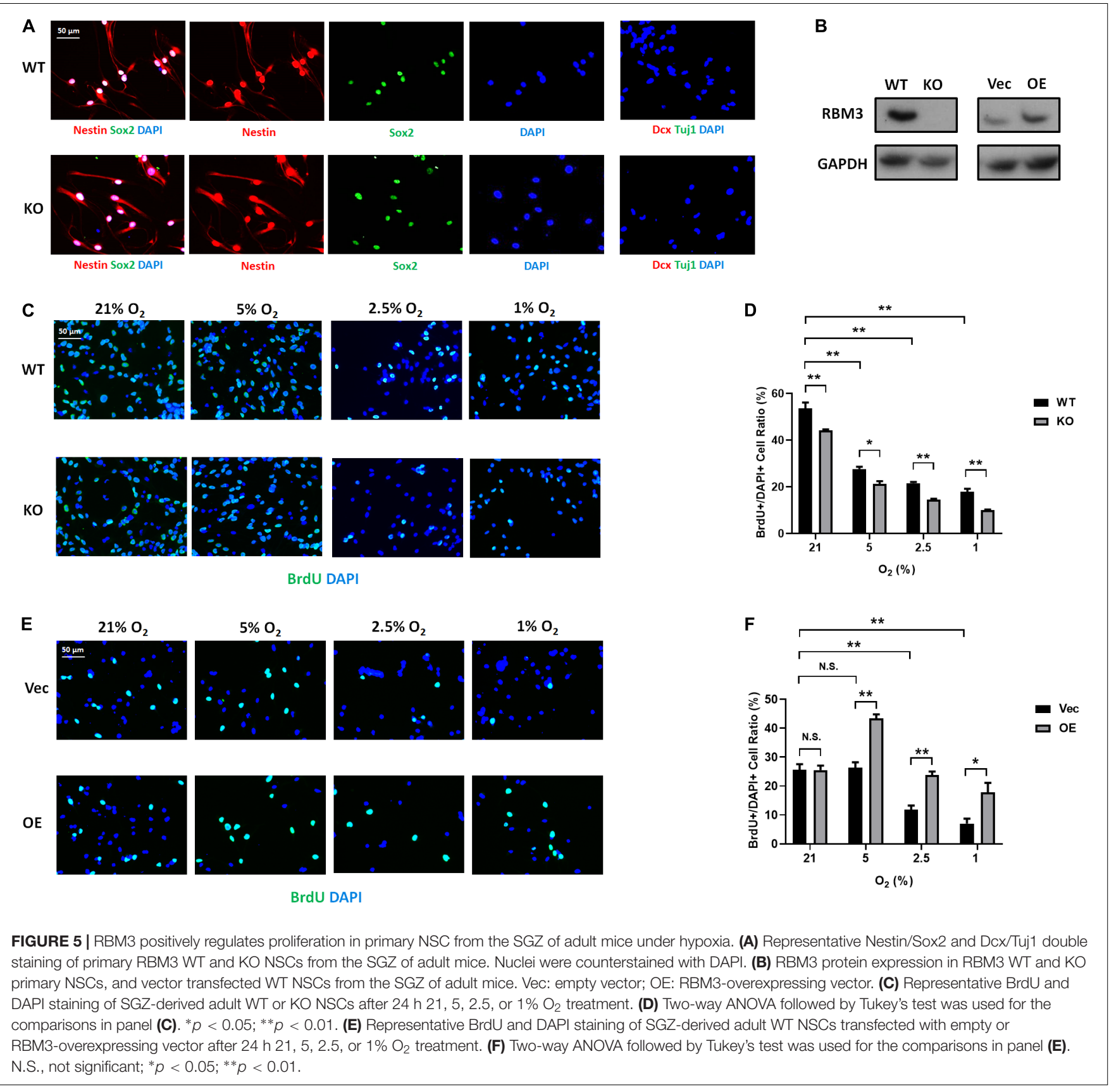

involved in mediating neuro-inflammation (Rajayer et al., 2013; Zhou et al., 2014), instead, RBM3 tends to be a safer target in clinical use.

Ischemic stroke and global HI injury are the most common cerebral hypoxic injuries, which induce irreversible damage to the brain from infants to adults (Huang and Castillo, 2008; Ekker et al., 2018). Although NSCs normally reside in hypoxic niche in physiological conditions, they can also migrate to injured regions for neuro-regeneration in these pathological conditions with moderate to severe hypoxia. Our recent study provides evidence that RBM3 behaves as a potential target to maintain NSC pool in HI conditions (Zhu et al., 2019). In addition, as the endogenous migrating NSCs are usually not sufficient to replace lost neurons by neurogenesis, exogenous transplantation of NSC is required for improved recovery not only by cell replacement, but also by multiple by-stand mechanisms (Kalladka and Muir, 2014; Vishwakarma et al., 2014; Huang and Zhang, 2019). When preparing exogenous NSCs in vitro, physiologically hypoxic pre-conditioning may benefit to mimic in vivo environment (Wakai et al., 2016), but can also produce disadvantageous effects, as hypoxia is a complex process. As the promotion of proliferation is largely mediated by hypoxia-inducible factor- $1 \alpha$ (HIF-1 $\alpha$ ) signaling (Mohyeldin et al., 2010; Li et al., 2014), RBM3 regulation is independent of HIF-1 $\alpha$ (Wellmann et al., 2004). 
Therefore, manipulating RBM3 expression opens a new avenue to increase viability, proliferation capacity, and multi-potency of cultured NSCs and probably other types of stem cells, which may improve therapeutic effects after transplantation.

\section{DATA AVAILABILITY STATEMENT}

All datasets generated for this study are available upon request to the corresponding author.

\section{ETHICS STATEMENT}

The animal experiment involved in this study was approved by the Cantonal Veterinary Office of Basel (License Number 2064). All the manipulations were executed according to the Ethical Principles and Guidelines for Experiments on Animals of the Swiss Academy of Medical Sciences and the Swiss Academy of Sciences.

\section{AUTHOR CONTRIBUTIONS}

$\mathrm{XZ}$ and SW designed the study, interpreted data, and wrote the manuscript. JY and TG performed all experiments in part assisted by AZ. RG and JK were involved in the interpretation and discussion of the results. All authors approved the manuscript.

\section{REFERENCES}

Bond, A. M., Ming, G. L., and Song, H. (2015). Adult mammalian neural stem cells and neurogenesis: five decades later. Cell Stem Cell 17, 385-395. doi: 10.1016/j.stem.2015.09.003

Chen, P., Yue, X., Xiong, H., Lu, X., and Ji, Z. (2019). RBM3 upregulates ARPC2 by binding the 3'UTR and contributes to breast cancer progression. Int. J. Oncol. 54, 1387-1397. doi: 10.3892/ijo.2019.4698

Chip, S., Zelmer, A., Ogunshola, O. O., Felderhoff-Mueser, U., Nitsch, C., Buhrer, C., et al. (2011). The RNA-binding protein RBM3 is involved in hypothermia induced neuroprotection. Neurobiol. Dis. 43, 388-396. doi: 10.1016/j.nbd.2011. 04.010

Clarke, L., and Van Der Kooy, D. (2009). Low oxygen enhances primitive and definitive neural stem cell colony formation by inhibiting distinct cell death pathways. Stem Cells 27, 1879-1886. doi: 10.1002/stem.96

Danno, S., Itoh, K., Matsuda, T., and Fujita, J. (2000). Decreased expression of mouse Rbm3, a cold-shock protein, in Sertoli cells of cryptorchid testis. Am. J. Pathol. 156, 1685-1692. doi: 10.1016/s0002-9440(10)65039-0

De Filippis, L., and Delia, D. (2011). Hypoxia in the regulation of neural stem cells. Cell Mol. Life Sci. 68, 2831-2844. doi: 10.1007/s00018-011-0723-5

Ekker, M. S., Boot, E. M., Singhal, A. B., Tan, K. S., Debette, S., Tuladhar, A. M., et al. (2018). Epidemiology, aetiology, and management of ischaemic stroke in young adults. Lancet Neurol. 17, 790-801. doi: 10.1016/S1474-4422(18)30233-3

Felfly, H., Zambon, A. C., Xue, J., Muotri, A., Zhou, D., Snyder, E. Y., et al. (2011). Severe Hypoxia: consequences to neural stem cells and neurons. J. Neurol. Res. 1:10.4021/jnr70w. doi: 10.4021/jnr70w

Francis, K. R., and Wei, L. (2010). Human embryonic stem cell neural differentiation and enhanced cell survival promoted by hypoxic preconditioning. Cell Death Dis. 1:e22. doi: 10.1038/cddis.2009.22

Fuentealba, L. C., Obernier, K., and Alvarez-Buylla, A. (2012). Adult neural stem cells bridge their niche. Cell Stem Cell 10, 698-708. doi: 10.1016/j.stem.2012. 05.012

\section{FUNDING}

This study was funded by the Swiss National Science Foundation (SNSF, 31003A_163305) and the China Scholarship Council (CSC, 201606170071).

\section{ACKNOWLEDGMENTS}

We sincerely thank Dr. Simone Keck and Janine Boegli (University of Basel, Switzerland) for the assistance of flow cytometry experiments.

\section{SUPPLEMENTARY MATERIAL}

The Supplementary Material for this article can be found online at: https://www.frontiersin.org/articles/10.3389/fcell.2019.00288/ full\#supplementary-material

TABLE S1 | Stability test of housekeeping genes under hypoxia for real-time PCR. The Ct values of six commonly used housekeeping genes, beta-actin (actb), glyceraldehyde 3-phosphate dehydrogenase (gapdh), ribosomal protein L13a (rp/13a), 45S pre-ribosomal RNA (rn45s), 28S ribosomal RNA (rn28s1) and alpha-tubulin-1 (tuba1), were measured by real-time PCR under 21, 5, 2.5, and $1 \%$ hypoxic conditions in $\mathrm{C} 17.2$ cells. Their stability was ranked by geNorm method (https://genorm.cmgg.be/). Actb shows the lowest $M$ value, indicating its highest stability under hypoxic conditions and thereby selected as reference gene in real-time PCR experiments.

Gage, F. H., and Temple, S. (2013). Neural stem cells: generating and regenerating the brain. Neuron 80, 588-601. doi: 10.1016/j.neuron.2013.10.037

Huang, B. Y., and Castillo, M. (2008). Hypoxic-ischemic brain injury: imaging findings from birth to adulthood. Radiographics 28, 417-439. doi: 10.1148/rg. 282075066

Huang, L., and Zhang, L. (2019). Neural stem cell therapies and hypoxic-ischemic brain injury. Prog. Neurobiol. 173, 1-17. doi: 10.1016/j.pneurobio.2018.05.004

Jackson, T. C., Manole, M. D., Kotermanski, S. E., Jackson, E. K., Clark, R. S., and Kochanek, P. M. (2015). Cold stress protein RBM3 responds to temperature change in an ultra-sensitive manner in young neurons. Neuroscience 305, 268-278. doi: 10.1016/j.neuroscience.2015.08.012

Kalladka, D., and Muir, K. W. (2014). Brain repair: cell therapy in stroke. Stem Cells Cloning 7, 31-44.

Lengronne, A., Pasero, P., Bensimon, A., and Schwob, E. (2001). Monitoring S phase progression globally and locally using BrdU incorporation in $\mathrm{TK}(+)$ yeast strains. Nucleic Acids Res. 29, 1433-1442. doi: 10.1093/nar/29.7. 1433

Li, L., Candelario, K. M., Thomas, K., Wang, R., Wright, K., Messier, A., et al. (2014). Hypoxia inducible factor-1alpha (HIF-1alpha) is required for neural stem cell maintenance and vascular stability in the adult mouse SVZ. J. Neurosci. 34, 16713-16719. doi: 10.1523/JNEUROSCI.4590-13.2014

Ludwig, P. E., Thankam, F. G., Patil, A. A., Chamczuk, A. J., and Agrawal, D. K. (2018). Brain injury and neural stem cells. Neural Regen. Res. 13, 7-18. doi: 10.4103/1673-5374.224361

Mas-Bargues, C., Sanz-Ros, J., Roman-Dominguez, A., Ingles, M., GimenoMallench, L., El Alami, M., et al. (2019). Relevance of oxygen concentration in stem cell culture for regenerative medicine. Int. J. Mol. Sci. 20:E1195. doi: 10.3390/ijms20051195

Matsuda, A., Ogawa, M., Yanai, H., Naka, D., Goto, A., Ao, T., et al. (2011). Generation of mice deficient in RNA-binding motif protein 3 (RBM3) and characterization of its role in innate immune responses and cell growth. Biochem. Biophys. Res. Commun. 411, 7-13. doi: 10.1016/j.bbrc.2011.06.038 
Mohyeldin, A., Garzon-Muvdi, T., and Quinones-Hinojosa, A. (2010). Oxygen in stem cell biology: a critical component of the stem cell niche. Cell Stem Cell 7, 150-161. doi: 10.1016/j.stem.2010.07.007

Panchision, D. M. (2009). The role of oxygen in regulating neural stem cells in development and disease. J. Cell Physiol. 220, 562-568. doi: 10.1002/jcp.21812

Pilotte, J., Cunningham, B. A., Edelman, G. M., and Vanderklish, P. W. (2009). Developmentally regulated expression of the cold-inducible RNA-binding motif protein 3 in euthermic rat brain. Brain Res. 1258, 12-24. doi: 10.1016/j. brainres.2008.12.050

Rajayer, S. R., Jacob, A., Yang, W. L., Zhou, M., Chaung, W., and Wang, P. (2013). Cold-inducible RNA-binding protein is an important mediator of alcoholinduced brain inflammation. PLoS One 8:e79430. doi: 10.1371/journal.pone. 0079430

Santilli, G., Lamorte, G., Carlessi, L., Ferrari, D., Rota Nodari, L., Binda, E., et al. (2010). Mild hypoxia enhances proliferation and multipotency of human neural stem cells. PLoS One 5:e8575. doi: 10.1371/journal.pone.0008575

Sureban, S. M., Ramalingam, S., Natarajan, G., May, R., Subramaniam, D., Bishnupuri, K. S., et al. (2008). Translation regulatory factor RBM3 is a protooncogene that prevents mitotic catastrophe. Oncogene 27, 4544-4556. doi: 10. 1038/onc. 2008.97

Ushio, A., and Eto, K. (2018). RBM3 expression is upregulated by NF-kappaB p65 activity, protecting cells from apoptosis, during mild hypothermia. J. Cell Biochem. 119, 5734-5749. doi: 10.1002/jcb.26757

Vieira, H. L., Alves, P. M., and Vercelli, A. (2011). Modulation of neuronal stem cell differentiation by hypoxia and reactive oxygen species. Prog. Neurobiol. 93, 444-455. doi: 10.1016/j.pneurobio.2011.01.007

Vishwakarma, S. K., Bardia, A., Tiwari, S. K., Paspala, S. A., and Khan, A. A. (2014). Current concept in neural regeneration research: NSCs isolation, characterization and transplantation in various neurodegenerative diseases and stroke: a review. J. Adv. Res. 5, 277-294. doi: 10.1016/j.jare.2013.04.005

Wakai, T., Narasimhan, P., Sakata, H., Wang, E., Yoshioka, H., Kinouchi, H., et al. (2016). Hypoxic preconditioning enhances neural stem cell transplantation therapy after intracerebral hemorrhage in mice. J. Cereb. Blood Flow Metab. 36, 2134-2145. doi: 10.1177/0271678x15613798

Wang, B., Jedlicka, S., and Cheng, X. (2014). Maintenance and neuronal cell differentiation of neural stem cells $\mathrm{C} 17.2$ correlated to medium availability sets design criteria in microfluidic systems. PLoS One 9:e109815. doi: 10.1371/ journal.pone.0109815

Wellmann, S., Bettkober, M., Zelmer, A., Seeger, K., Faigle, M., Eltzschig, H. K., et al. (2008). Hypoxia upregulates the histone demethylase JMJD1A via HIF-1. Biochem. Biophys. Res. Commun. 372, 892-897. doi: 10.1016/j.bbrc.2008.05.150

Wellmann, S., Buhrer, C., Moderegger, E., Zelmer, A., Kirschner, R., Koehne, P., et al. (2004). Oxygen-regulated expression of the RNA-binding proteins RBM3 and CIRP by a HIF-1-independent mechanism. J. Cell Sci. 117, 1785-1794. doi: $10.1242 /$ jcs. 01026
Wellmann, S., Truss, M., Bruder, E., Tornillo, L., Zelmer, A., Seeger, K., et al. (2010). The RNA-binding protein RBM3 is required for cell proliferation and protects against serum deprivation-induced cell death. Pediatr. Res. 67, 35-41. doi: 10.1203/PDR.0b013e3181c13326

Xia, W., Su, L., and Jiao, J. (2018). Cold-induced protein RBM3 orchestrates neurogenesis via modulating Yap mRNA stability in cold stress. J. Cell Biol. 217, 3464-3479. doi: 10.1083/jcb.201801143

Yang, H. J., Ju, F., Guo, X. X., Ma, S. P., Wang, L., Cheng, B. F., et al. (2017). RNA-binding protein RBM3 prevents NO-induced apoptosis in human neuroblastoma cells by modulating p38 signaling and miR-143. Sci. Rep. 7:41738. doi: 10.1038/srep41738

Yuan, L. L., Guan, Y. J., Ma, D. D., and Du, H. M. (2015). Optimal concentration and time window for proliferation and differentiation of neural stem cells from embryonic cerebral cortex: 5\% oxygen preconditioning for 72 hours. Neural Regen. Res. 10, 1516-1522. doi: 10.4103/1673-5374.165526

Zhang, Q., Wang, Y. Z., Zhang, W., Chen, X., Wang, J., Chen, J., et al. (2017). Involvement of cold inducible RNA-binding protein in severe hypoxia-induced growth arrest of neural stem cells in vitro. Mol. Neurobiol. 54, 2143-2153. doi: 10.1007/s12035-016-9761-1

Zhou, M., Yang, W. L., Ji, Y., Qiang, X., and Wang, P. (2014). Cold-inducible RNAbinding protein mediates neuroinflammation in cerebral ischemia. Biochim. Biophys. Acta 1840, 2253-2261. doi: 10.1016/j.bbagen.2014.02.027

Zhu, X., Buhrer, C., and Wellmann, S. (2016). Cold-inducible proteins CIRP and RBM3, a unique couple with activities far beyond the cold. Cell Mol. Life Sci. 73, 3839-3859. doi: 10.1007/s00018-016-2253-7

Zhu, X., Yan, J., Bregere, C., Zelmer, A., Goerne, T., Kapfhammer, J. P., et al. (2019). RBM3 promotes neurogenesis in a niche-dependent manner via IMP2-IGF2 signaling pathway after hypoxic-ischemic brain injury. Nat. Commun. 10:3983. doi: 10.1038/s41467-019-11870-x

Zhuang, R. J., Ma, J., Shi, X., Ju, F., Ma, S. P., Wang, L., et al. (2017). Cold-inducible protein RBM3 protects UV irradiation-induced apoptosis in neuroblastoma cells by affecting p38 and JNK pathways and Bcl2 family proteins. J. Mol. Neurosci. 63, 142-151. doi: 10.1007/s12031-017-0964-3

Conflict of Interest: The authors declare that the research was conducted in the absence of any commercial or financial relationships that could be construed as a potential conflict of interest.

Copyright (c) 2019 Yan, Goerne, Zelmer, Guzman, Kapfhammer, Wellmann and Zhu. This is an open-access article distributed under the terms of the Creative Commons Attribution License (CC BY). The use, distribution or reproduction in other forums is permitted, provided the original author(s) and the copyright owner(s) are credited and that the original publication in this journal is cited, in accordance with accepted academic practice. No use, distribution or reproduction is permitted which does not comply with these terms. 